\title{
Nitric oxide and atrial natriuretic factor stimulate cGMP-dependent membrane insertion of aquaporin 2 in renal epithelial cells
}

\author{
Richard Bouley, ${ }^{1}$ Sylvie Breton, ${ }^{1,2}$ Tian-xiao Sun, ${ }^{1}$ Margaret McLaughlin, ${ }^{1}$ \\ Ndona N. Nsumu, ${ }^{1}$ Herbert Y. Lin, ${ }^{1,2}$ Dennis A. Ausiello, ${ }^{1,2}$ and Dennis Brown ${ }^{1,3}$ \\ ${ }^{1}$ Program in Membrane Biology and Renal Unit, Massachusetts General Hospital, Charlestown, Massachusetts, USA \\ ${ }^{2}$ Department of Medicine, and \\ ${ }^{3}$ Department of Pathology, Harvard Medical School, Boston, Massachusetts, USA
}

Address correspondence to: Dennis Brown, Renal Unit and Program in Membrane Biology, Room 8207, Massachusetts General Hospital East, 149 13th Street, Charlestown, Massachusetts 02129, USA.

Phone: (617) 726-5665; Fax: (617) 726-5669; E-mail: brown@receptor.mgh.harvard.edu.

Received for publication February 11, 2000, and accepted in revised form September 19, 2000.

In collecting duct principal cells, aquaporin 2 (AQP2) is shuttled from intracellular vesicles to the plasma membrane upon vasopressin (VP) stimulation. VP activates adenylyl cyclase, increases intracellular cAMP, activating protein kinase A (PKA) to phosphorylate $\mathrm{AQP} 2$ on the $\mathrm{COOH}$-terminal residue, serine 256. Using rat kidney slices and LLC-PK1 cells stably expressing AQP2 (LLC-AQP2 cells), we now show that AQP2 trafficking can be stimulated by cAMP-independent pathways. In these systems, the nitric oxide (NO) donors sodium nitroprusside (SNP) and NONOate and the NO synthase substrate L-arginine mimicked the effect of VP, stimulating relocation of AQP2 from cytoplasmic vesicles to the plasma membrane. Unlike VP, these other agents did not increase intracellular cAMP. However, SNP increased intracellular cGMP, and exogenous cGMP stimulated AQP2-membrane insertion. Atrial natriuretic factor, which signals via cGMP, also stimulated AQP2 translocation. The VP and SNP effects were blocked by the kinase inhibitor H89. SNP did not stimulate membrane insertion of AQP2 in LLC-PK1 cells expressing the phosphorylation-deficient mutant 256SerAla-AQP2, indicating that phosphorylation of Ser256 is required for signaling. Both PKA and cGMP-dependent protein kinase G phosphorylated AQP2 on this COOH-terminal residue in vitro. These results demonstrate a novel, cAMP-independent and cGMP-dependent pathway for AQP2 membrane insertion in renal epithelial cells.

J. Clin. Invest. 106:1115-1126 (2000).

\section{Introduction}

Nitric oxide (NO) is an important mediator of numerous physiological functions in several organs and cell types $(1,2)$. In the kidney, three isoforms of NO synthase (NOS) have been detected by RT-PCR and in situ hybridization (3-6). Endothelial NOS (eNOS) mRNA is located in glomeruli, preglomerular vasculature, proximal tubules, thick ascending limbs, and collecting ducts in normal rats, and mRNA for inducible NOS (iNOS) is expressed in proximal tubules, medullary thick ascending limbs, and cortical outer- and inner-medullary collecting ducts. Neuronal NOS (nNOS) was found in thick ascending limbs of Henle, vasa recta, glomeruli, and renal nerves, as well as in collecting ducts where it was detectable in principal cells, but not in intercalated cells. While NOS is implicated in modulating renal blood flow and in the regulation of sodium handling (7), some data suggest that NO may affect the renal urinary concentrating mechanism. The NG-nitro-L-arginine methyl ester (L-NAME), a NOS inhibitor, induced both diuresis and natriuresis in anesthetized rats (8). In water-deprived rats, an increase of eNOS mRNA expression accompanied a reduction in urine output (9).
A considerable amount of work has shown that aquaporin (AQP) water channels are important for urinary concentration and body fluid homeostasis. AQP1 is present in both apical and basolateral plasma membranes of proximal tubules and thin descending limbs of Henle (10, 11). AQP3 and AQP4 are located constitutively on the basolateral plasma membrane of principal cells of the collecting duct (12-15). AQP2 is expressed in collecting duct principal cells, where its plasma-membrane expression is stimulated by the antidiuretic hormone vasopressin (VP) (16-21). The cellular and molecular mechanisms underlying this hormonally induced, regulated trafficking of AQP2 are the subject of intense current investigation. VP binding to its receptor (V2R) activates adenylyl cyclase and increases cAMP levels in principal cells, which in turn leads to AQP2 phosphorylation by protein kinase A (PKA) $(22,23)$. Phosphorylation of AQP2 at serine-256 in the cytoplasmic carboxyl-terminus is necessary for the VP-regulated movement of AQP2-containing vesicles to the plasma membrane $(24,25)$.

Because NOS is expressed in principal cells and the NOS signal transduction pathway also involves the activation of specific protein kinases, but via a cGMP-depend- 
ent pathway, we examined the effect on AQP2 trafficking of $\mathrm{NO}$ and atrial natriuretic factor (ANF), both of which increase cGMP in collecting ducts (26). Our data show that activation of a cGMP-mediated signaling pathway results in the membrane insertion of AQP2 in collecting duct principal cells, as well as in AQP2-transfected LLCPK1 epithelial cells (LLC-AQP2 cells).

\section{Methods}

Chemicals and reagents. Lysine vasopressin (LVP), arginine vasopressin (AVP), sodium nitroprusside (SNP), L-arginine, forskolin, atrial natriuretic factor (ANF), 3-isobutyl-1-methylxanthine (IBMX), H89, and 8-bromo guanosine 3',5'-cyclic monophosphate (8-BRcGMP) were purchased from Sigma (St. Louis, Missouri, USA). All cell-culture reagents, including Geneticin, medium, and FBS were purchased from GIBCO BRL (Grand Island, New York, USA). LVP, the porcine antidiuretic hormone, was used to stimulate pig kidney-derived LLC-PK1 cells, while AVP was used on rat tissues. For simplicity, both forms of the hormone will be referred to as VP in the remainder of the text.

The overexpression vector pET-41a (which allows the production of glutathione-S-transferase [GST] fusion proteins) and thrombin cleavage kits were obtained from Novagen (Madison, Wisconsin, USA). High-fidelity Pfu DNA polymerase was from Stratagene (La Jolla, California, USA). The Escherichia coli expression strain BL21-SI was from GIBCO BRL. Complete proteaseinhibitor cocktail was purchased from Roche Molecular Biochemicals (Mannheim, Germany) and glutathione-Sepharose 4B was from Amersham Pharmacia Biotech (Piscataway, New Jersey, USA). Ni-TA-Superflow was from QIAGEN (Santa Clarita, California, USA). $\left[\gamma^{32} \mathrm{P}\right]$ ATP with a specific activity of 5,000 $\mathrm{Ci} / \mathrm{mmol}$ was purchased from NEN Life Science Products (Boston, Massachusetts, USA). PKA and protein kinase $\mathrm{G}$ (PKG) catalytic subunits were obtained from Promega Corp. (Madison, Wisconsin, USA), and NuPAGE gels were from Invitrogen (Carlsbad, California, USA). The "specific" PKA and PKG inhibitors KT5720 and KT5823, respectively, were from Calbiochem-Novabiochem (San Diego, California, USA).

Kidney tissue slice preparation. Thin slices of kidney were prepared for in vitro studies as described previously (27). Male Sprague-Dawley rats were anesthetized with sodium pentobarbital, and the kidneys were perfused briefly through the abdominal aorta with HBSS pH 7.4 at $37^{\circ} \mathrm{C}$ equilibrated with $5 \% \mathrm{CO}_{2} / 95 \% \mathrm{O}_{2}$. When the kidneys were cleared of blood (about 1 minute), they were removed, and thin slices (approximately $0.5 \mathrm{~mm}$ ) were quickly cut using a Stadie-Riggs slicer (Thomas Scientific, Swedesboro, New Jersey, USA). The slices were preincubated at $37^{\circ} \mathrm{C}$ for 15 minutes in equilibrated HBSS. Either VP (10 nM) plus forskolin (10 $\mu \mathrm{M})$, freshly diluted SNP $(1 \mathrm{mM})$, L-arginine $(10 \mathrm{mM})$, or ANF $(1 \mu \mathrm{M})$ were then added for various periods of time up to 15 minutes. To determine the location of AQP2 at the beginning and the end of the incubation period, some slices were fixed before the addition of drugs or agonists and others after 15 minutes in HBSS. After incubation, all slices were fixed by immersion in periodate-lysine paraformaldehyde (PLP) fixative for 5-6 hours at room temperature. Slices were then rinsed several times in PBS $(10 \mathrm{mM}$ sodium phosphate buffer containing $0.9 \% \mathrm{NaCl}, \mathrm{pH} 7.4)$.

Immunocytochemistry. Kidney slices were cryoprotected in PBS containing 30\% sucrose for 2 hours at room temperature. Slices were mounted on a cutting block, covered with OCT compound 4583 (Tissue-Tek; Miles Inc., Elkhart, Indiana, USA) and cryosections ( $4 \mu \mathrm{m}$ thick) were cut using a Reichert Frigocut microtome (Leica Microsystems, Deerfield, Illinois, USA). Sections were picked up on Fisher Superfrost Plus slides (Fisher Scientific, Philadelphia, Pennsylvania, USA) and stored at $4^{\circ} \mathrm{C}$ before immunofluorescence staining. After rehydration in PBS for 5 minutes, sections were incubated in PBS containing 1\% BSA for 15 minutes to block nonspecific staining. Sections were incubated with a previously characterized antiserum raised against the second extracellular loop of AQP2 (28) (diluted 1:100 in PBS) for 1.5 hours at room temperature, followed by two washes of 5 minutes each in high-salt PBS (containing $2.7 \% \mathrm{NaCl}$ ) to reduce background staining and one final wash in PBS. Sections were then incubated with goat anti-rabbit IgG conjugated to FITC at a final concentration of $5 \mu \mathrm{g} / \mathrm{ml}$ (Kirkegaard \& Perry Labs, Gaithersburg, Maryland, USA) for 1 hour at room temperature and then rinsed as for the primary Ab. Sections were mounted in Vectashield (Vector Labs, Burlingame, California, USA) diluted 2:1 in 0.1 M Tris- $\mathrm{HCl}, \mathrm{pH}$ 8.0, and were examined using a Bio-Rad Radiance 2000 confocal laser scanning microscope (Bio-Rad, Hercules, California, USA). Final images were imported into Adobe Photoshop (Adobe Systems Inc., Mountain View, California, USA) as TIFF files and were printed in CMYK color using an Epson 600 inkjet printer.

Quantification oflabeling. Sections of control, SNP-, and $\mathrm{VP} /$ forskolin-treated kidney slices from different animals (see legends to figures for $n$ values) were stained with anti-AQP2 Ab's. One set of measurements was performed on slices incubated for 15 minutes with agonists, and another set of measurements was performed to quantify the time course $(0,2,5,10$, and 15 minutes) of $\mathrm{SNP}$ and $\mathrm{VP} /$ forskolin action on $\mathrm{AQP} 2$ redistribution. Images were collected using a Bio-Rad Radiance 2000 confocal microscope using the same aperture size. Gain and/or laser power were set so that the brightest regions of the images were just below saturation level using the "setcolor" feature of the Bio-Rad Lasersharp software. After collection, images were imported into IP Lab Spectrum (Scanalytics, Vianna, Virginia, USA) as TIFF files for analysis. The segmentation function was used to obtain total cell area, excluding the nucleus, by setting the minimum pixel intensity for inclusion at a low value so that the entire cytoplasm of stained cells was highlighted. The total area occupied by the highlighted pixels was taken as total principal cell area. Intercalated cells 
and nuclei were not highlighted with this procedure. Next, the minimum threshold value for highlighting was increased until the highlighted pixels corresponded in each image to the AQP2-associated fluorescence pattern observed in the original, nonmanipulated image. This part of the procedure was subjective, but by switching back and forth between the raw image and the threshold image, an accurate representation of the fluorescencestaining pattern could be reproduced reliably. The total area of the highlighted pixels was once more obtained for each cell or group of cells. Between 10 and 40 measurements were taken for each sample, and samples from at least three different controls and experimental sets of tissue were taken. Results are expressed as mean plus or minus SEM of the percentage of total cell area occupied by the fluorescence signal. This value represents the spread of AQP2-associated fluorescence throughout the cells. A larger value indicates more diffuse, cytoplasmic fluorescence, while a smaller value indicates that the fluorescence was more concentrated in a smaller region of the cell, in this case, the apical pole of the cell. The quantification was performed only on cells in which both apical and basolateral membrane domains were visible.

Immunogold electron microscopy of tissue slices. For the detection of AQP2 in principal cells using electron microscopy, the same incubation and fixation conditions described above were used. After thorough rinsing, tissue slices from control, 15-minute SNP-, and 15minute $\mathrm{VP} /$ forskolin-treated samples were cut into thinner sections $(50 \mu \mathrm{m})$ using a Vibratome (Ted Pella Inc., Redding, California, USA). These final slices were incubated overnight with an anti-AQP2 Ab (1:100 dilution) raised against an external epitope of AQP2 (28) without any detergent permeabilization step. This procedure ensured that only cell-surface (i.e., plasma membrane) AQP2 would be detected. After rinsing, the slices were incubated overnight in goat anti-rabbit IgG coupled to colloidal gold particles (10 nm diameter) (Ted Pella Inc.). After further rinsing, slices were fixed in $1 \%$ glutaraldehyde, osmified, dehydrated, and flatembedded in Epon 812. Thin sections were stained with uranyl acetate and lead citrate and examined using a Philips CM10 electron microscope (Philips Electronics Inc., Mahwah, New Jersey, USA).

Cell culture. The production and growth of LLC-PK1 cells that were stably transfected with c-myc epitopetagged AQP2 cDNA (LLC-AQP2 cells) have been described previously (29). LLC-PK1 cells stably transfected with a Ser256 to Ala AQP2 mutant cDNA (LLCS256A cells) have also been described previously (24). Cells were passaged every 4 days at 1:10 dilution and maintained in 10\% FBS/DMEM.

Immunofluorescence on cell cultures. Cells were grown on glass coverslips (Bellco Glass, Vineland, New Jersey, USA) to approximately $60 \%$ confluence and rinsed with incubation buffer (PBS, $\mathrm{pH} 7.4$, containing 0.9 $\mathrm{mM} \mathrm{CaCl}_{2}, 0.9 \mathrm{mM} \mathrm{MgCl}_{2}, 3.5 \mathrm{mM} \mathrm{KCl}$, and $1 \mathrm{mg} / \mathrm{ml}$ glucose). Cells were then treated with $10 \mathrm{nM}$ VP, $1 \mathrm{mM}$ SNP, $10 \mathrm{mM}$ NONOate, $0.1 \mathrm{mM}$ 8-BRcGMP, $10 \mathrm{mM}$
L-arginine, or $1 \mu \mathrm{M}$ ANF for 10 minutes. LLC-AQP2 cells were also pretreated for 30 minutes with H89 (30 $\mu \mathrm{M})$, a PKA and PKG inhibitor, before incubation with VP or SNP for 10 minutes. Cells were fixed with $4 \%$ paraformaldehyde and 5\% sucrose in PBS for 20 minutes. They were washed three times in PBS and incubated with an anti-c-myc mAb (30) in PBS at room temperature for 1 hour. The cells were then incubated with goat anti-mouse IgG conjugated to indocarbocyanine (CY3) $(2 \mu \mathrm{g} / \mathrm{ml}$; Jackson ImmunoResearch, West Grove, Pennsylvania, USA) for 1 hour, washed, and mounted in Vectashield. The cells were examined using either a Nikon Eclipse 800 microscope or a BioRad Radiance 2000 confocal microscope. For final printing, the positive staining was pseudocolored in green, and images were printed using a Tektronix Phaser 440 dye sublimation printer (Tektronix, Wilsonville, Oregon, USA).

cAMP and cGMP assay. LLC-AQP2 cells were plated onto 96-well plates and grown to confluence. The cAMP and cGMP assays were performed with the BioTrak kit (Amersham Pharmacia Biotech). Briefly, cells were pretreated with $1 \mathrm{mM}$ IBMX followed by forskolin $(10 \mu \mathrm{M})$ or SNP (1 mM) for 10 minutes. The cells were then lysed, centrifuged, and the supernatant was mixed with rabbit anti-cAMP Ab or rabbit anti-cGMP Ab. Aliquots were added to 96-well plates precoated with donkey anti-rabbit Ab. Then cAMP or cGMP-peroxidase conjugate was added for 1 hour, the plates were washed, and the peroxidase substrate was added. Sulfuric acid was added to terminate the reaction. The OD at $450 \mathrm{~nm}$ was read on a plate reader. The ODs were compared with standard curves (0-200 fmol/well of cAMP) or (0-512 $\mathrm{fmol} /$ well of cGMP). This is a competition assay in which higher OD values reflect the presence of less cyclic nucleotide in the original cell supernatant. Each intracellular cAMP and cGMP accumulation assay was performed in triplicate.

Cloning, expression, and purification of a GST-AQP2 $\mathrm{COOH}$-tail fusion protein. An AQP2 COOH-tail fusion protein was developed for use as a substrate for PKAand PKG-mediated in vitro phosphorylation. A hexaHis tag was introduced onto the $\mathrm{NH}_{2}$-terminus of the AQP2 COOH-tail (L223-A271) coding sequence by PCR using Pfu polymerase with rat AQP2 cDNA as a template. The PCR product was cloned into the expression vector $\mathrm{pET}-41$, which is designed for the production of GST-fusion proteins, as a BamHI/HindIII fragment and was sequenced to rule out PCR errors. The E. coli strain BL21-SI was transformed with the construct, and bacterial production was carried out using a 2-1 bench-top Virtis (New York, New York, USA) fermentation system. The final density of E. coli cells was between $\mathrm{OD}_{600} 20-30$ at the time of harvesting.

Protein extraction and purification were performed according to the manufacturer's instructions. Briefly, bacteria were resuspended in lysis buffer $(50 \mathrm{mM}$ Tris$\mathrm{HCl}, \mathrm{pH}$ 8.0, $100 \mathrm{mM} \mathrm{NaCl}, 1 \mathrm{mM}$ EDTA) containing Complete protease inhibitor (Roche Molecular Bio- 


\section{Figure 1}

Confocal microscope images of tissue slices showing AQP2 in principal cells from inner stripe (IS) collecting ducts. A kidney from one rat was cut into thin slices and incubated in vitro with or without agonists for 15 minutes before fixation by immersion, sectioning, and immunostaining to detect AQP2. (a) Control, buffer alone; (b) $10 \mathrm{nM} V \mathrm{P}$ plus $10 \mu \mathrm{M}$ forskolin; (c) 1 mM SNP; (d) 10 mM L-arginine. Under control conditions (a), AQP2 is located in numerous intracellular vesicles and shows no apparent membrane staining. AVP plus forskolin, SNP, and L-arginine all induce a marked redistribution of AQP2 to the apical plasma membrane. Intercalated cells in the collecting duct epithelium are unstained. This result is representative of three sets of experiments using a different animal each time. Bar, $10 \mu \mathrm{m}$.
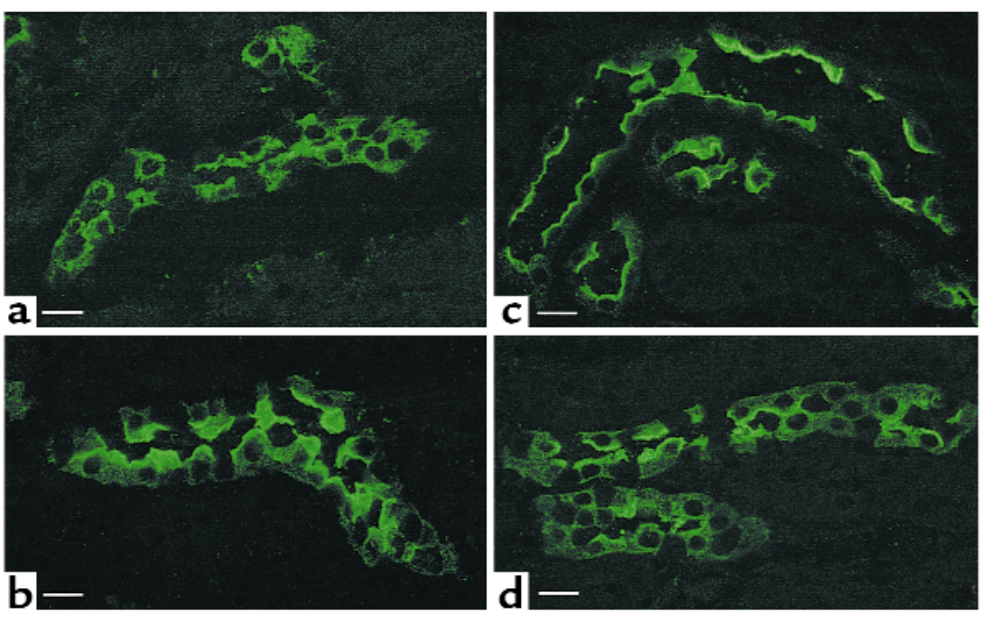

chemicals) and incubated at room temperature with $0.1 \mathrm{mg} / \mathrm{ml}$ lysozyme for 30 minutes followed by $1 \%$ Triton X-100. After centrifugation, the supernatant was clarified by $0.5 \%$ polyethylenimine precipitation, and the fusion protein was absorbed onto glutathioneSepharose beads. After release from the beads using a thrombin cleavage buffer, the partially purified AQP2 tail was further purified using a Ni-NTA superflow column. The $6 \mathrm{xHis}$-tagged AQP2 tail was eluted from the column in PBS/400 mM imidazole, and the final product was dialyzed against PBS and stored at $-80^{\circ} \mathrm{C}$. The purity of the product was confirmed by SDS PAGE (see Figure 11a). The sequence of the thrombin cleavage product is:

GSTAIGMKETAAAKFERQHMDSPDLGTGGGSGDDDDKSPMGYRGSMGHHHHHHLFPSAKSLQERLVAVLKGLEPDTDWEEREVRRRQSVELHSPQSLQRGSKA (the AQP2 COOH-tail sequence is in bold).

In vitro phosphorylation assay. To assess the phosphorylation of AQP2 by PKG in vitro, $4 \mu \mathrm{M}$ purified AQP2 COOH-tail peptide was mixed with $4 \mathrm{nM}$ PKG catalytic subunit in a total volume of $10 \mu \mathrm{l}$ reaction buffer containing $40 \mathrm{mM}$ Tris- $\mathrm{HCl}, \mathrm{pH}$ 7.4, $20 \mathrm{mM} \mathrm{MgOAc}$, $10 \mu \mathrm{M}$ ATP, $10 \mu \mathrm{Ci}\left[\gamma_{-}{ }^{32} \mathrm{P}\right] \mathrm{ATP}, 2 \mu \mathrm{M}$ cGMP, and was incubated at $25^{\circ} \mathrm{C}$ for 10 minutes. The reaction was stopped by boiling the samples in SDS-PAGE sample buffer. To determine PKA phosphorylation, 4 nM PKA catalytic subunit was included in the incubation buffer, and cAMP replaced cGMP. For inhibition experiments, the catalytic subunits were heat inactivated before use, and other incubations were performed in the presence of the kinase inhibitors KT5720 (PKA) or KT5823 (PKG) (31) at a final concentration of $10 \mu \mathrm{M}$. Samples were resolved on a $12 \%$ NuPage gel. Protein phosphorylation was visualized using autoradiography. Quantification of the bands was performed using NIH Image software (NIH, Bethesda, Maryland, USA).

Statistical analysis. Data are expressed as mean plus or minus SEM. Statistical analyses were made using the Student $t$ test or ANOVA when applicable. Differences were considered significant at $P$ values less than 0.05 .

\section{Results}

NO stimulates AQP2 translocation in collecting duct principal cells

Confocal immunofluorescence microscopy. In the inner stripe of the outer medulla, AQP2 showed a diffuse pattern of staining in the cytoplasm of collecting duct principal cells under control conditions (Figure 1a). AQP2 was located predominantly on cytoplasmic vesicles in cells fixed before the addition of any agonists (not shown), as well as after a further incubation period in buffer alone (Figure 1a). Adjacent intercalated cells were not labeled. The kidneys used to prepare these slices had been removed from the animal, rapidly cut into thin slices at room temperature, and placed in oxygenated Hank's buffered saline at $37^{\circ} \mathrm{C}$. Low temperatures were avoided because our previous data showed that exposure of kidney slices to cold buffer solution results in microtubule depolymerization and mislocalization of some membrane proteins, especially during the subsequent rewarming period (27). Control tissues that were fixed before agonist addition had been VP free for about 15-20 minutes before the start of the experiment. Thus, the control samples represent the distribution of AQP2 after VP washout.

As expected, the distribution of AQP2 was significantly modified by the addition of VP $(10 \mathrm{nM})$ plus forskolin $(10 \mu \mathrm{M})$ to the buffer for 15 minutes. AQP2 was relocated toward the apical pole of most cells after 15 minutes (Figure 1b). A similar marked relocation of AQP2 was also found in collecting ducts exposed to SNP $(1 \mathrm{mM})$ for 15 minutes (Figure 1c). Furthermore, translocation of AQP2 to the apical plasma membrane was induced by the addition of L-arginine to the incubation medium (Figure 1d). It thus appears that NO (produced directly from SNP or indirectly from L-arginine) stimulates the insertion of AQP2 water channels into the apical membrane of collecting duct principal cells. The stimulatory effect of SNP on AQP2 membrane insertion was observed in the inner stripe of the outer medulla. While VP stimulated the membrane insertion of AQP2 in all regions, the effect of SNP on $\mathrm{AQP} 2$ distribution was 


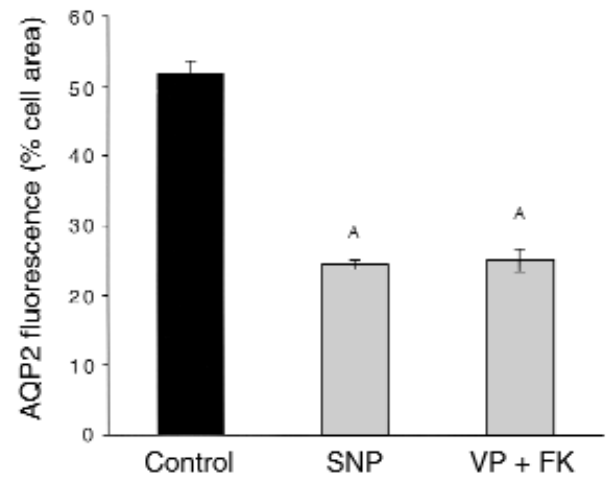

much less obvious in the cortex, the outer stripe of the inner medulla, and in the initial portion of the innermedullary collecting duct, and no effect at all was detected in papillary collecting ducts (not shown).

The effect of VP/forskolin and SNP on AQP2 distribution in the inner stripe was quantified by using densitometry on images collected by laser confocal microscopy. Three separate experiments were quantified, using kidney slices from three different rats. In this quantification, the total area occupied by AQP2 fluorescence reflects the cellular distribution of the protein. The larger the area occupied, the more diffusely scattered is the protein within the cells examined. Movement to the membrane is reflected by a decrease in the total area of fluorescence, since AQP2containing vesicles within each cell migrate into a smaller apical band of fluorescence staining. The data in Figure 2 show that both SNP and VP caused a highly significant reduction in the area of the cell occupied by AQP2. Taken together with the staining patterns shown in Figure 1, these data indicate that SNP stimu- lates AQP2 movement from cytoplasmic vesicles to the cell surface in a manner that resembles the known effect of VP on this process.

A time-course experiment showed that some relocation of AQP2 to the plasma membrane was detectable as soon as 2 minutes after SNP or VP/forskolin addition to kidney slices (Figure 3, a and c), although a significant amount of intracellular staining was still observed at this early time point. The stimulatory effect of these agonists was more pronounced after 5 minutes and reached an apparent maximum after 10 minutes of stimulation (Figures 3, b and d). A quantitative evaluation (Figure 4) of the staining patterns was performed on confocal images, as described above. In control slices fixed after 15 minutes of preincubation ( $t=0$ in Figure 4$)$, AQP2 is located mainly in intracellular vesicles. The cytosolic distribution was slightly more evident after a further 15 minute incubation in vitro ( $t=15$ minutes in Figure 4). A progressive relocation of AQP2 to the apical membrane was observed upon addition of agonists, with a maximum effect seen after 10 minutes. After 15 minutes
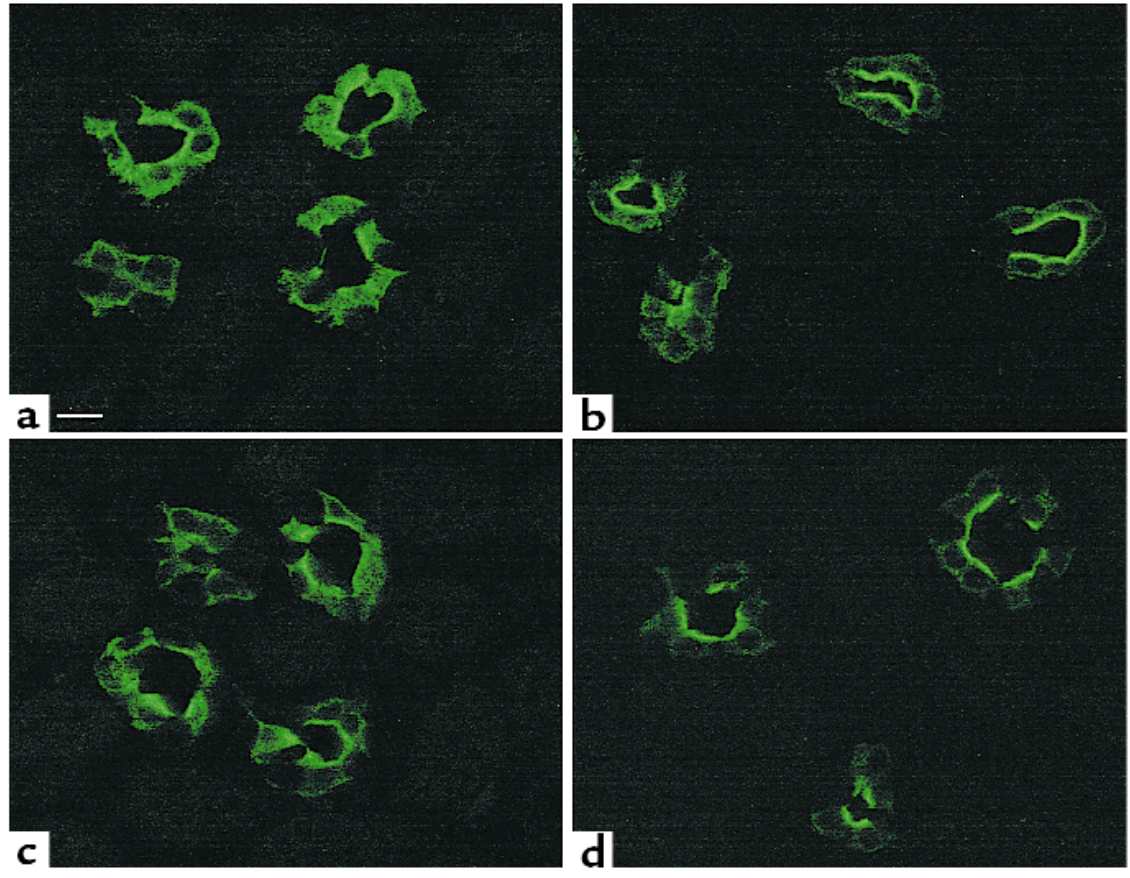

\section{Figure 3}

Confocal images showing the time course of $\mathrm{VP} /$ forskolin-induced and SNP-induced (10 $\mu \mathrm{M})$ translocation of AQP2 to the apical plasma membrane in collecting duct (inner stripe) principal cells. (a) Collecting duct stained 2 minutes after addition of $\mathrm{VP} /$ forskolin; (b) stained 10 minutes after addition of $\mathrm{VP} /$ forskolin; (c) stained 2 minutes after addition of SNP; (d) stained 10 minutes after addition of SNP. AQP2 is distributed intracellularly before agonist addition, but shows a progressive translocation to the apical cell surface during incubation with VP/forskolin and SNP (see Figure 4 for a quantification of the time course of agonist-induced AQP2 translocation). Some apical staining is already detectable 2 minutes after agonist addition, but a significant amount of intracellular staining is still present at this early time point $(\mathbf{a}, \mathbf{c})$. The maximum effect is seen after 10 minutes of treatment, when most of the staining is located on the apical membrane (b,d). Bar, $10 \mu \mathrm{m}$. 


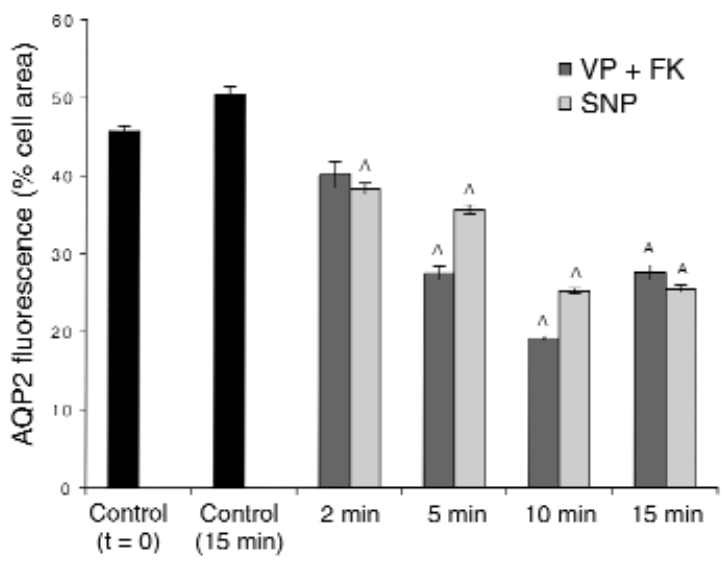

Figure 4

Quantification of the time course of AQP2 translocation to the apical plasma membrane of principal cells after VP/forskolin and SNP stimulation. The quantification of AQP2-membrane insertion was performed in the same way as described in Figure 2. AQP2 was distributed throughout the cytoplasm before addition of agonists, and this distribution was slightly (but not significantly) more pronounced after a further 15 minutes of in vitro incubation in the absence of agonists. A redistribution of AQP2 was already detectable 2 minutes after VP/forskolin or SNP addition and became progressively more marked after 5 and 10 minutes of treatment. After 15 minutes, the SNP effect was similar to the 10-minute response, while the effect of $\mathrm{VP} /$ forskolin was somewhat less pronounced after 15 minutes of treatment, although the difference was not statistically significant compared with the 10 -minute time point. ${ }^{A} P<0.05$ compared with control values (controls, both $t=0$ and $t=15$ minutes).

of treatment, a slight reversal of the $\mathrm{VP} /$ forskolin effect was observed, but the apical staining remained significantly greater than in the control, nonstimulated tissue. In contrast, the effect of SNP was not significantly diminished after 15 minutes of stimulation.

Immunogold electron microscopy. To confirm that the tight apical band of fluorescence seen in principal cells after stimulation represented true apical membrane localization of AQP2, immunogold electron microscopy was performed. Figure 5 shows that after 15 minutes of SNP (Figure $5 \mathrm{a}$ ) or VP/forskolin (Figure $5 \mathrm{~b}$ ) treatment, many gold particles were found on the external surface of the apical membrane of principal cells. Adjacent intercalated cells were completely unstained, consistent with the absence of AQP2 from this cell type. Control, unstimulated tissues had very little apical staining, although occasional gold-particle clusters could be found (Figure 5c). Because the labeling was performed using an antiexternal epitope $\mathrm{Ab}$, and because the tissues were not permeabilized, this staining pattern indicates that after SNP and VP/forskolin stimulation, AQP2 is inserted into the apical plasma membrane of principal cells in the tissue slice preparation.

NO stimulates AQP2-membrane insertion in LLC-AQP2 cells

The effect of SNP on the localization of AQP2 was examined by confocal microscopy in LLC-AQP2 cells, in which VP-regulated trafficking of AQP2 has been extensively characterized $(29,32,33)$. In nonstimulated cells, AQP2 was predominantly localized on intracellular vesicles (Figure 6a). After exposure of the cells to SNP $(1 \mathrm{mM})$ for 10 minutes, the localization of AQP2 shifted from an intracellular vesicle pattern to a predominantly plasma membrane staining (Figure 6b). This effect was indistinguishable from that produced by forskolin $(10 \mu \mathrm{M})$ (Figure 6c)
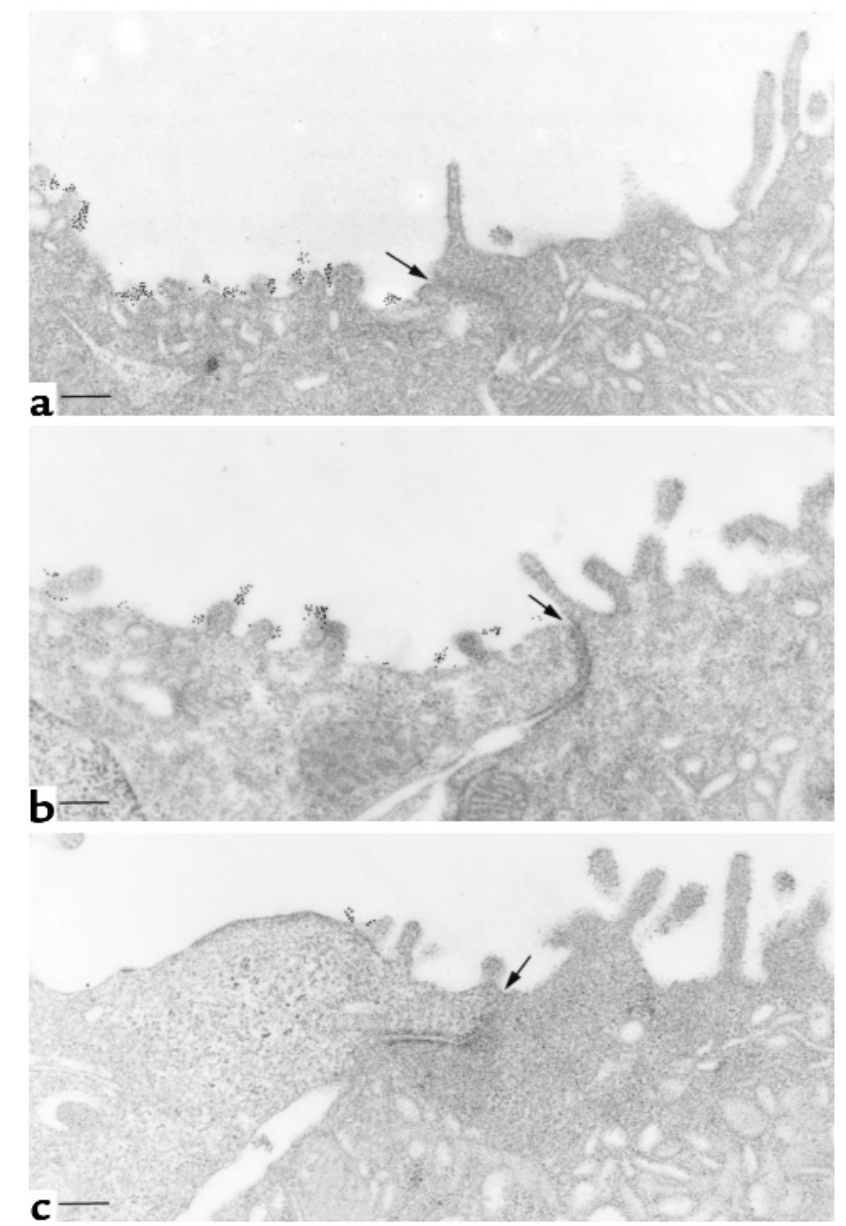

Figure 5

Immunogold electron microscopy showing apical plasma membrane insertion of AQP2 induced by VP/forskolin and SNP treatment of kidney slices. AQP2 was localized in nonpermeabilized tissues with an Ab against an external epitope of AQP2. (a) SNP treatment for 15 minutes. Abundant gold particles, representing AQP2 antigenic sites, are located on the apical plasma membrane of a principal cell (left). An adjacent intercalated cell (right) is unlabeled. (b) Apical plasma membrane localization of gold labeling for AQP2 in a principal cell after VP/forskolin treatment. The adjacent intercalated cell is unlabeled. (c) Control tissue incubated for 15 minutes in the absence of agonist. Very few gold particles are seen on the apical membrane, indicating that most of the AQP2 is inside the cell in this condition, supporting the confocal data shown in Figures 1 and 3 . In all figures, most of the gold particles are on the external surface of the apical membrane, consistent with the use of an $\mathrm{Ab}$ raised against an external epitope of AQP2. The position of the cell junction between the principal cell (left) and the intercalated cell (right) is indicated with an arrow in each figure. Bar, $1 \mu \mathrm{m}$. 
or VP (not shown). A similar effect on AQP2 redistribution was seen after treatment of LLC-AQP2 cells with a different NO donor, diethylamine NONOate (not shown), and with the NO synthase substrate L-arginine (not shown). Our previous immunogold studies on LLC-AQP2 cells have shown clearly that the peripheral AQP2 staining pattern described here after agonist stimulation represents plasma-membrane staining and is not due to vesicles in close proximity to the plasma membrane (32). Thus, as for the in vitro tissue slices, $\mathrm{NO}$ and L-arginine stimulate the translocation of AQP2 from intracellular vesicles to the cell surface of AQP2-transfected LLC-PK1 cells.

SNP increases intracellular cGMP but not cAMP in LLC-AQP2 cells

Because translocation of AQP2 to the plasma membrane occurs after an increase in cytosolic cAMP, we tested the effect of SNP on intracellular cAMP levels in LLC-AQP2 cells. Incubation of these cells with VP (10 $\mathrm{nM})$ for 10 minutes caused a large increase in intracellular cAMP, an effect that was mimicked by $10 \mu \mathrm{M}$ forskolin (Figure 7). In contrast, incubation of LLCAQP2 cells with $1 \mathrm{mM}$ SNP for 10 minutes did not increase intracellular cAMP above basal levels. However, SNP treatment did increase the intracellular content of cGMP to more than 30 times greater than the basal level. Neither VP nor forskolin had any effect on intracellular cGMP levels. These data suggest that the effect of NO on AQP2 translocation is cAMP independent.

AQP2 translocation is stimulated by cGMP in LLC-AQP2 cells

Since cGMP, but not cAMP, levels were increased by SNP in parallel with translocation of AQP2 to the cell surface, the effect of the membrane-permeant analogue of cGMP, 8-BRcGMP, on AQP2 trafficking was examined. As shown in Figure 6d, 8-BRcGMP (0.1 mM) alone for 10 minutes was sufficient to stimulate a marked accumulation of AQP2 at the plasma membrane of LLC-AQP2 cells. Thus, AQP2 trafficking can be induced not only by cAMP, but also by cGMP, consistent with the stimulatory effect of SNP on intracellular cGMP levels and on AQP2 translocation.

ANF stimulates AQP2 translocation in principal cells and LLC-AQP2 cells

We next tested the effect of a physiologically relevant agonist, ANF, on AQP2 translocation in our experimental systems. As shown in Figure 8a, $10 \mu \mathrm{M}$ ANF had a dramatic effect on the intracellular distribution of AQP2 in principal cells of collecting ducts from the inner stripe of the outer medulla. As for the other agonists tested, ANF treatment resulted in a clear apical localization of AQP2 in principal cells. The effect was equally obvious in LLC-AQP2 cells. Figure 8b shows a strong plasmamembrane staining for AQP2 in LLC-AQP2 cells that were exposed to $10 \mu \mathrm{M}$ ANF for 10 minutes. The quantitative data in Figure 9 show that ANF caused a highly significant reduction in the area of collecting duct principal cells occupied by AQP2, as shown above for SNP and VP/forskolin (compare with Figure 2). Thus, a physiological mediator that acts through cGMP has an effect similar to SNP, indicating that the observed results are probably not due to the elevation of intracellular $\mathrm{NO} / \mathrm{cGMP}$ through a nonphysiological intervention.

The serine256 residue of AQP2 is necessary for NO stimulation of AQP2 translocation

Serine 256 in the cytoplasmic COOH-terminal tail of AQP2 is known to be the site of PKA-induced phosphorylation. Phosphorylation of S256 is necessary for AQP2 accumulation at the cell surface after VP stimulation $(25,32)$. However, the S256 consensus sequence is also a target motif for phosphorylation of proteins by another kinase, PKG, which is stimulated by cGMP.
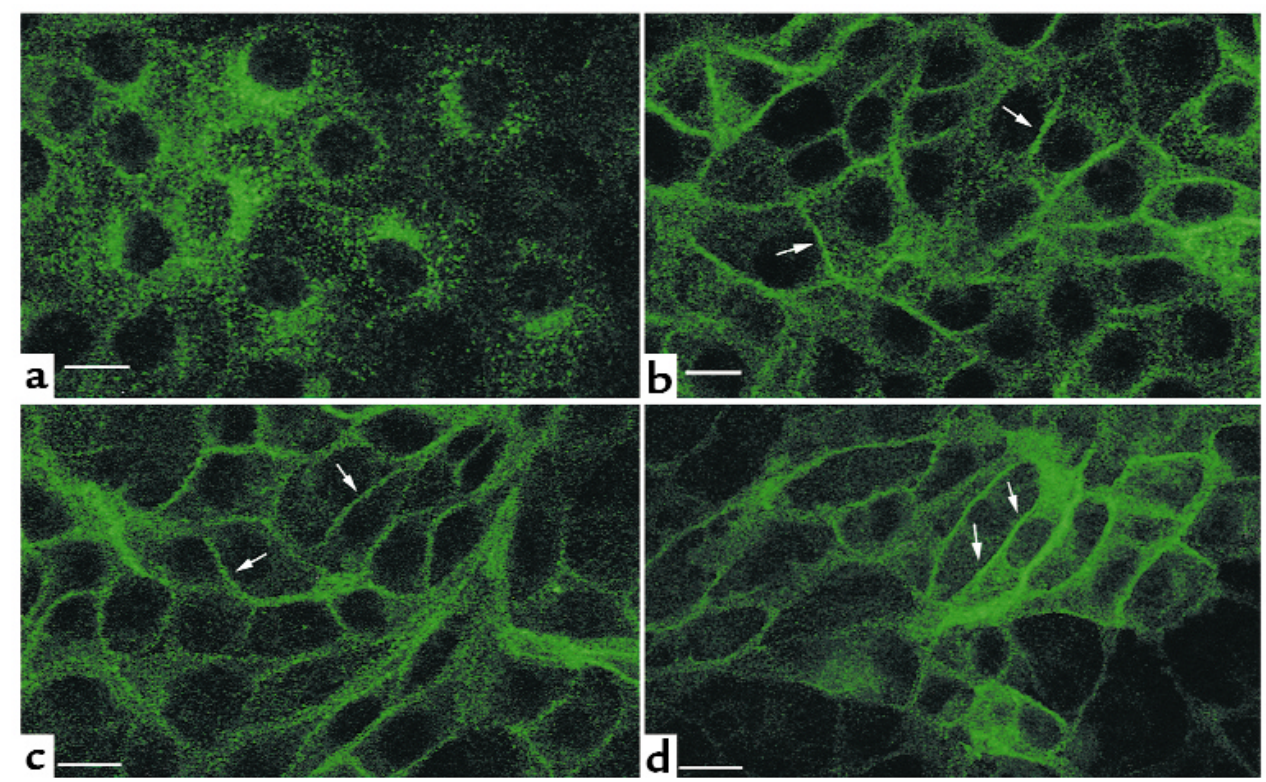

\section{Figure 6}

Immunofluorescence localization of AQP2 in LLC-AQP2 cells showing that SNP, forskolin, and dibutyryl cGMP stimulate AQP2 translocation to the plasma membrane. In nonstimulated cells (a), the c-myc Ab stains many cytoplasmic vesicles, but the plasma membrane is virtually unstained. In contrast, AQP2 shows a plasma membrane localization (arrows) after incubation for 10 minutes with $1 \mathrm{mM}$ SNP (b), $10 \mu \mathrm{M}$ forskolin (c), and cGMP (d). Bar, $5 \mu \mathrm{m}$. 


\section{a}

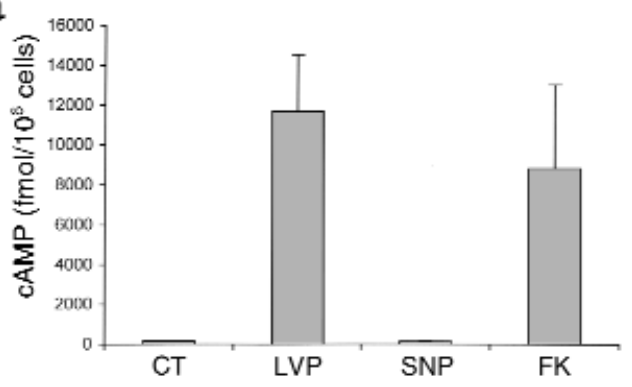

b

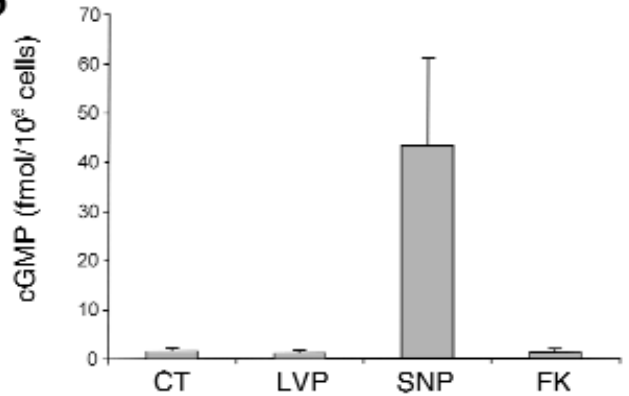

Figure 7

Effect of VP and SNP treatment on intracellular levels of cAMP and cGMP in LLC-AQP2 cells. Cells were incubated for 10 minutes with 10 $\mathrm{nM} V \mathrm{P}, 1 \mathrm{mM}$ SNP, or $10 \mu \mathrm{M}$ forskolin (FK). After incubation, the cells were solubilized and supernatants were used to measure the intracellu$\operatorname{lar} \operatorname{cAMP}(\mathbf{a})$ and $\operatorname{cGMP}(\mathbf{b})$ using an ELISA assay. Each point represents the mean \pm SEM of triplicate determinations. Similar data were obtained in two more separate experiments.

To test the involvement of this sequence in the response to SNP, we first examined the effect of H89, an inhibitor of both PKA and PKG on the SNP stimulation of AQP2 translocation. Figure 10 shows that the SNP-induced translocation of AQP2 to the plasma membrane was completely inhibited by $\mathrm{H} 89$ treatment of the cells (30 $\mu \mathrm{M}$ for 30 minutes) (Figure 10a). We have shown previously that $\mathrm{H} 89$ also inhibits the VPinduced translocation of AQP2 to the plasma membrane in these cells (24). Interestingly, and as shown previously, H89 caused AQP2 to become concentrated in a perinuclear patch, possibly the Golgi or the transGolgi network through which AQP2 recycles during its intracellular trafficking (33). Thus, both the AVP and SNP stimulation of AQP2 translocation involve protein kinase activity. We next used LLC-S256A cells that were stably transfected with an AQP2 construct in which serine 256 had been replaced by alanine (S256A-AQP2). We and others have shown previously that AVP does not stimulate AQP2 trafficking in LLC-S256A cells (25, 32 ). Figure $10 \mathrm{~b}$ shows the localization of S256A-AQP2 in these cells after SNP treatment. S256A-AQP2 was still localized on intracellular vesicles; it did not translocate to the cell surface. Therefore, the S256 residue is necessary for both the VP- and SNP-induced AQP2 translocation to the cell surface, presumably through a phosphorylation step.

PKG phosphorylates AQP2 in vitro

Using a GST-AQP2 COOH-tail fusion protein as a substrate, we assessed the ability of purified PKG to phosphorylate the AQP2 $\mathrm{COOH}$ tail. The purity of the recombinant AQP2 COOH tail is shown in Figure 11a, in which a single band at about $12-13 \mathrm{kDa}$, the theoretical size of the recombinant peptide, is present. PKA was used as a positive control. As shown in Figure 11b, a strong phosphorylation of the AQP2 $\mathrm{COOH}$ tail was seen using both PKG and PKA. This phosphorylation was inhibited using KT5823 (a PKG inhibitor) and KT5720 (a PKA inhibitor), respectively, and no band was present when heat-inactivated catalytic subunits were used. However, significant inhibition was also found when the two "specific" inhibitors were reversed, although the inhibition was somewhat weaker in this cross-inhibition assay. The relative intensities of the phosphorylated bands are shown in the lower part of Figure 11b. For this reason, we could not use the KT family of inhibitors in our cell-culture model as a convincing means of dissecting PKA versus PKG effects. No phosphorylation was detected when GST alone, with no AQP2 tail, was used in the assay (not shown). These data demonstrate that both PKA and PKG can phosphorylate AQP2 in our in vitro assay, supporting the idea that PKG could also perform this function in the intact cell.

\section{Discussion}

NO plays an important role in preserving renal blood flow during states of peripheral vasoconstriction such as angiotensin excess or dehydration (7). It affects both endothelial cells and the juxtaglomerular apparatus to preserve regional blood flow and to decrease fluid and sodium reabsorption in the proximal convoluted tubule

\section{Figure 8}

ANF stimulates plasma membrane insertion of AQP2 in kidney epithelial cells. Rat kidney slices and LLC-AQP2 cells were incubated with $10 \mu \mathrm{M}$ ANF for 10 minutes, followed by localization of AQP2 by indirect immunofluorescence. Under these conditions, AQP2 was located in the apical plasma membrane of principal cells in innerstripe collecting ducts (a) and in the basolateral plasma membrane of LLC-AQP2 cells in culture (b). Bar, $5 \mu \mathrm{m}$.
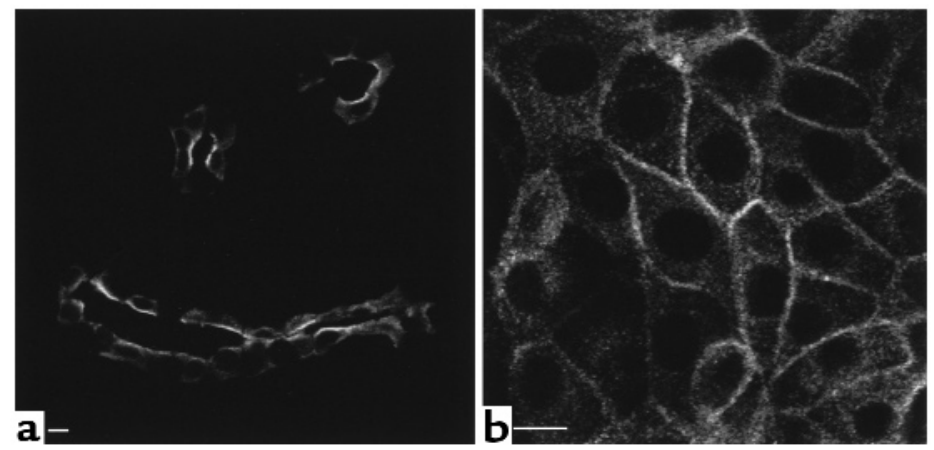


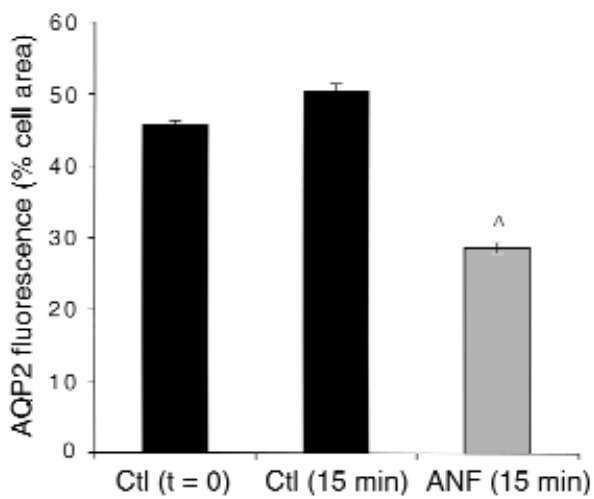

\section{Figure 9}

Quantification of the effect of ANF on AQP2 redistribution in collecting duct (inner stripe) principal cells. Total principal cell area (excluding the nucleus) and the area occupied by AQP2 fluorescence were determined using IP Lab Spectrum software on confocal microscope images such as those shown in Figure 8a. Results are expressed as the percentage of total cell area occupied by AQP2 fluorescence. ANF treatment ( $10 \mu \mathrm{M}$ ANF for 10 minutes) induces a marked reduction in the area of AQP2 fluorescence, indicating it causes the AQP2 staining to become concentrated in a smaller region of the cell, i.e., the apical pole. ${ }^{A} P<0.05$ compared with the control values. This ANF experiment was performed in parallel with the time course study shown in Figure 4 , and the controls ( $t=0$ and $t=15$ minutes) are, therefore, the same.

(34). However, other aspects of the role of NO in renal physiology are not entirely clear, although several observations suggest that $\mathrm{NO}$ might play a role in collecting duct fluid and electrolyte transport. During dehydration, NOS mRNA and protein are increased in the kidney (9). One isoform of NOS, nNOS, is expressed specifically in principal cells of the collecting duct (35). Furthermore, the $\beta 2$-subunit of guanylate cyclase is present only in collecting duct principal cells (36). Our present data show that $\mathrm{NO}$ induces the cytoplasm-to-membrane translocation of AQP2 water channels both in collecting duct principal cells and in AQP2-transfected LLC-PK1 epithelial cells (LLC-AQP2 cells). This cAMP-independent trafficking pathway, activated by different $\mathrm{NO}$ donors, including SNP, L-arginine, and NONOate, could play a role in urinary concentration and body fluid homeostasis.

ANF also has a "VP-like" effect on AQP2 distribution both in collecting duct principal cells and in LLC-AQP2 epithelial cells. Both cell types have ANF receptors, and ANF increases cGMP in these cells (37). cGMP-specific phosphodiesterase $\mathrm{V}$ is also present in the collecting duct (38), and the PDE V inhibitor zaprinast increases cGMP in response to ANF when applied to isolated collecting ducts of rats with nephrotic syndrome (39). The effect of ANF (through an increase in cGMP) on ion and fluid transport by the collecting duct is controversial and seems also to depend on the precise collecting duct segment that is examined. Some reports show that it decreases both sodium reabsorption and VP-induced water flow in collecting ducts $(40,41)$, whereas others do not confirm these inhibitory effects $(42,43)$. Furthermore, the effect of SNP on the cortical collecting duct has been reported to be both cGMP-independent (44) and cGMP-dependent (45). SNP does not increase cGMP in the inner medullary collecting duct (46), which is consistent with our present findings that it does not cause any detectable redistribution of AQP2 in this portion of the collecting duct. Thus, the interplay between ANF- and VP-regulated signaling in the collecting duct is complex and remains unresolved.

A considerable amount of work on the renal concentrating mechanism has shown that VP stimulates the movement of AQP2 from cytoplasmic vesicles to the plasma membrane in the collecting duct principal cells during states of dehydration. The increase in apical membrane water permeability caused by AQP2 insertion allows osmotic equilibration between the luminal fluid (urine) and the hypertonic medullary interstitium to occur, resulting in urinary concentration. This process has been shown to depend upon an increase in cytosolic cAMP, activation of PKA, and phosphorylation of AQP2 at residue S256 in the cytoplasmic tail of the protein (22-25). Our present data suggest that a similar chain of events can be initiated by $\mathrm{NO}$ in the absence of any detectable increase in cytoplasmic cAMP levels. However, NO does increase cytosolic cGMP, and we show here that 8-BRcGMP can also stimulate the membrane insertion of AQP2 in cultured epithelial cells.

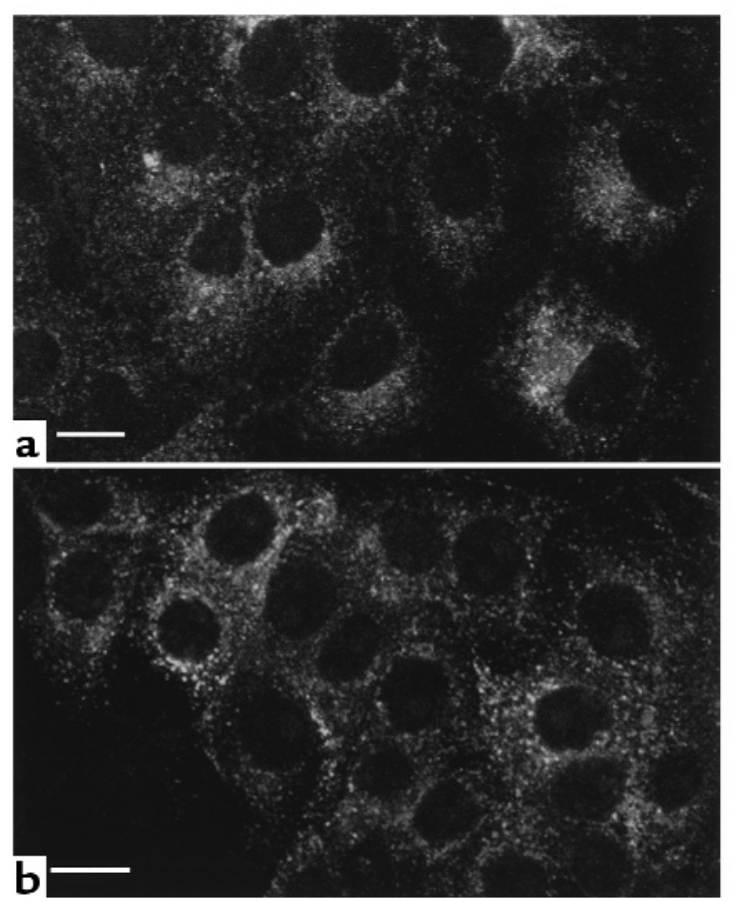

\section{Figure 10}

SNP does not induce AQP2 relocalization in LLC-AQP2 cells pretreated for 30 minutes with the PKA-inhibitor $\mathrm{H} 89(30 \mu \mathrm{M})$ or in cells expressing the S256A-mutant form of AQP2. Using Ab's against the c-myc epitope tag on the AQP2 $\mathrm{COOH}$-terminus, AQP2 was detected on perinuclear intracellular vesicles under basal conditions. This location did not change with SNP in $\mathrm{H} 89$ treated cells (a) or in cells expressing the S256A mutated form of AQP2 (b), in contrast to the effect of SNP in cells expressing the wild-type AQP2 (see Figure 6b). Bar, $5 \mu \mathrm{m}$. 


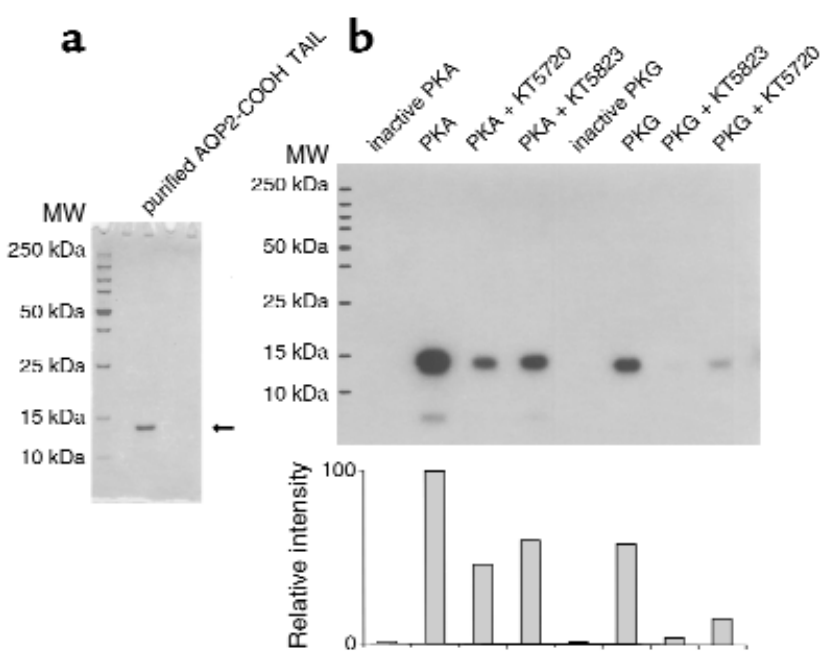

Figure 11

In vitro phosphorylation of the AQP2 $\mathrm{COOH}$ tail by PKA and PKG. Purified AQP2 $\mathrm{COOH}$ tail was used as a substrate for the phosphorylation assay. The AQP2 $\mathrm{COOH}$ tail was purified and runs as a single band at $13 \mathrm{kDa}$ on SDS-PAGE (a). Phosphorylation of this recombinant protein (b). It is phosphorylated by both PKA and PKG, and the phosphorylation is partially inhibited by $10 \mu \mathrm{M}$ KT5720 and KT5823, respectively. However, these "specific" reagents also inhibit phosphorylation in crossover assays, although somewhat less effectively than when they are used to inhibit their "specific" target (PKA for KT5720 and PKG for KT5823). No AQP2 was phosphorylated when the PKA and PKG catalytic subunits were heat inactivated before inclusion in the assays (inactive PKA and inactive PKG). Neither PKA nor PKG phosphorylated the fusion protein in the absence of the AQP2 $\mathrm{COOH}$ tail (not shown). A quantification (by NIH Image software) of the degree of phosphorylation is shown in the lower panel of $\mathbf{b}$.

Similar to the VP-stimulated process, cGMP-stimulated AQP2 trafficking to the cell surface is dependent upon the presence of the S256 phosphorylation consensus site in the cytoplasmic tail of AQP2 $(24,25)$. No effect of SNP on the intracellular localization of AQP2 was seen in S256A-AQP2-transfected cells. This suggests that AQP2 phosphorylation at S256 is required for cGMP-stimulated AQP2 trafficking, but whether the final phosphorylation step is through PKA or the cGMP-activated kinase PKG remains unknown. Our data show that PKG can directly phosphorylate AQP2 in vitro, but it is also conceivable that PKG activates PKA in vivo, which subsequently results in the phosphorylation of AQP2. While the kinase inhibitor H89 prevents SNP-induced AQP2 translocation (as well as AVP-induced AQP2 translocation), this inhibitor blocks the action of both PKA and PKG. The use of the more specific KT family of inhibitors theoretically could allow further dissection of this process; the relative $\mathrm{K}_{\mathrm{m}} \mathrm{S}$ of KT5720 and KT5823 for PKA and PKG, respectively, are only about 25 -fold different (31), and in vitro assays show considerable overlap in their specificities. While KT5720 inhibits VP-induced AQP2 translocation in LLC-AQP2 cells and KT5823 inhibits
SNP-induced AQP2 translocation, we have been unable to convincingly show an agonist-specific, differential effect of these two compounds on AQP2 trafficking in LLC-AQP2 cells (data not shown). Whatever the final mechanism of activation, our data provide strong evidence for a cAMP-independent pathway of AQP2 membrane insertion both in principal cells and in AQP2-transfected epithelial cells.

The mechanism by which AQP2 phosphorylation results in plasma membrane localization of AQP2 also remains to be elucidated. The increase of $\mathrm{AQP} 2$ at the cell surface could be due to an increase in exocytosis, a decrease in endocytosis, or a combination of both. We have reported previously that the apical distribution of AQP2 in principal cells in situ is disrupted by colchicine (18). In addition, cold treatment of kidney slices, which results in microtubule disruption, inhibits the VPinduced apical membrane insertion of AQP2 (27). It is possible that the microtubule-dependent movement of vesicles carrying AQP2 to the plasma membrane is stimulated by phosphorylation, perhaps by allowing more efficient interaction with microtubule motors such as the dynein ATPase, which can be found associated with AQP2-containing vesicles (47).

Some evidence for VP-independent (possibly, therefore, cAMP-independent) urinary concentration has been obtained both in humans and in experimental animal models. Patients with congenital nephrogenic diabetes insipidus (NDI) are treated with mild diuretics such as amiloride and hydrochlorothiazide, which

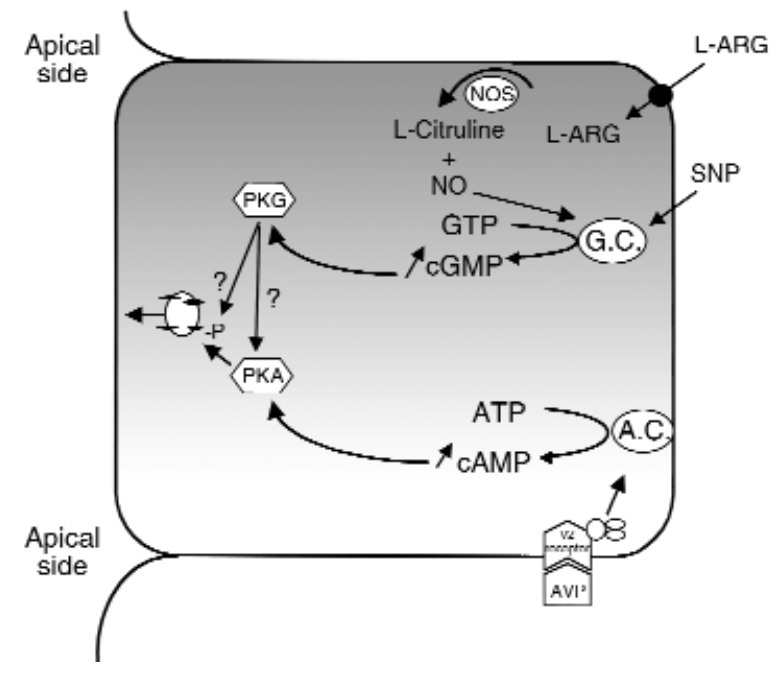

Figure 12

In current models of VP action, adenylate cyclase is stimulated by the interaction of VP with its receptor (V2R), and intracellular cAMP increases. PKA is activated, resulting in AQP2 phosphorylation on serine 256, followed by AQP2 translocation from vesicles to the cell surface. Our present data suggest an alternative pathway of AQP2 translocation. SNP (and ANF) activates guanylate cyclase resulting in increased cytosolic cGMP and PKG activation. Whether PKG directly phosphorylates AQP2 in intact cells (as it can in vitro - see Figure 11), or whether it activates PKA (either directly or indirectly), which in turn phosphorylates AQP2, remains unknown. 
is presumed to decrease the filtered load and lead to an increase in proximal reabsorption of the filtered fraction of water (48). However, not all patients respond well to diuretic therapy, and those who respond only partially may be the $10 \%$ of patients with mutations in their AQP2 gene and not the $90 \%$ with mutations in their V2R gene (49). Since diuretic therapy also induces a mild state of dehydration, it is possible that the dehydration increases water reabsorption in patients with normal AQP2 by an increase in NOS. In the Brattleboro rat, which has central diabetes insipidus and normal renal V2R and AQP2, there is evidence for an AVP-independent mechanism of urinary concentration and water-channel translocation in collecting ducts that has been related to oxytocin action on the V2R (50-52), but a potential role for $\mathrm{NO}$ in this response should now be examined. Interestingly, a report showing a hydroosmotic effect of cGMP on toad (Bufo arenarum) urinary bladders was published almost three decades ago (53), although studies using other species failed to detect such an effect $(54,55)$.

Our working model (Figure 12) is that NO (or ANF) causes an increase in intracellular cGMP, stimulates a kinase (either PKG and/or PKA) in a cAMP-independent manner, which then activates the intracellular trafficking system in renal epithelial cells to allow AQP2 to move to the cell surface. This result raises many important questions that can be addressed in future studies. What proportion of water reabsorption during dehydration is mediated by NO compared with AVP? What is the physiological significance of the observation that NO affects only outer medullary but not papillary AQP2 movement? Previous studies examining the effect of ANF/NO on epithelial water permeability have not focussed on this specific tubule segment. Of considerable interest is the possibility of using this new information to develop novel therapeutic strategies for the treatment of NDI, which up to now have focused on the VP-receptor/cAMP-intracellular signaling cascade.

\section{Acknowledgments}

This work was funded by NIH grants DK-38452 (D. Brown and S. Breton) and DK-19406 (D.A. Ausiello). H.Y. Lin is the recipient of an NIH KO8 award (DK02716). R. Bouley and T.-x. Sun are funded by fellowships from the National Kidney Foundation. R. Bouley was also supported by a fellowship from the Heart and Stroke Foundation of Canada.

1. Gross, S.S., and Wolin, M.S. 1995. Nitric oxide: pathophysiological mechanisms. Annu. Rev. Physiol. 57:737-769.

2. Bredt, D.S., and Snyder, S.H. 1994. Nitric oxide: a physiologic messenger molecule. Annu. Rev. Biochem. 63:175-195.

3. Ahn, K.Y., Mohaupt, M.G., Madsen, K.M., and Kone, B.C. 1994. In situ hybridization localization of mRNA encoding inducible nitric oxide synthase in rat kidney. Am. J. Physiol. 267:F748-F757.

4. Mohaupt, M.G., et al. 1994. Differential expression and induction of mRNAs encoding two inducible nitric oxide synthases in rat kidney. Kidney Int. 46:653-665.

5. Terada, Y., Tomita, K., Nonoguchi, H., and Marumo, F. 1992. Polymerase chain reaction localization of constitutive nitric oxide synthase and soluble guanylate cyclase messenger RNAs in microdissected rat nephron segments. J. Clin. Invest. 90:659-665.

6. Ujiie, K., Yuen, J., Hogarth, L., Danziger, R., and Star, R.A. 1994. Localization and regulation of endothelial NO synthase mRNA expression in rat kidney. Am. J. Physiol. 267:F296-F302.

7. Braam, B. 1999. Renal endothelial and macula densa NOS: integrated response to changes in extracellular fluid volume. Am. J. Physiol. 276:R1551-R1561.

8. Li, Q., Bowmer, C.J., and Yates, M.S. 1994. Diuretic effect of NG-nitro-Larginine methyl ester in the rat. J. Pharm. Pharmacol. 46:510-512.

9. Shin, Q., et al. 1999. Increased nitric oxide synthase mRNA expression in the renal medulla of water-deprived rats. Kidney Int. 56:2191-2202.

10. Nielsen, S., Smith, B.L., Christensen, E.I., Knepper, M.A., and Agre, P. 1993. CHIP28 water channels are localized in constitutively water-permeable segments of the nephron. J. Cell Biol. 120:371-383.

11. Sabolic, I., et al. 1992. Localization of the CHIP28 water channel in rat kidney. Am. J. Physiol. 263:C1225-C1233.

12. Ecelbarger, C.A., et al. 1995. Aquaporin-3 water channel localization and regulation in rat kidney. Am. J. Physiol. 269:F663-F672.

13. Frigeri, A., et al. 1995. Localization of MIWC and GLIP water channel homologs in neuromuscular, epithelial and glandular tissues. J. Cell Sci. 108:2993-3002.

14. Ishibashi, K., et al. 1994. Molecular cloning and expression of a member of the aquaporin family with permeability to glycerol and urea in addition to water expressed at the basolateral membrane of kidney collecting duct cells. Proc. Natl. Acad. Sci. USA. 91:6269-6273.

15. Terris, J., Ecelbarger, C.A., Marples, D., Knepper, M.A., and Nielsen, S. 1995. Distribution of aquaporin-4 water channel expression within rat kidney. Am. J. Physiol. 269:F775-F785.

16. Fushimi, K., et al. 1993. Cloning and expression of apical membrane water channel of rat kidney collecting tubule. Nature. 361:549-552.

17. Nielsen, S., DiGiovanni, S.R., Christensen, E.I., Knepper, M.A., and Harris, H.W. 1993. Cellular and subcellular immunolocalization of vasopressin-regulated water channel in rat kidney. Proc. Natl. Acad. Sci. USA. 90:11663-11667.

18. Sabolic, I., Katsura, T., Verbavatz, J.M., and Brown, D. 1995. The AQP2 water channel: effect of vasopressin treatment, microtubule disruption, and distribution in neonatal rats. J. Membr. Biol. 143:165-175.

19. Nielsen, S., et al. 1995. Vasopressin increases water permeability of kidney collecting duct by inducing translocation of aquaporin-CD water channels to plasma membrane. Proc. Natl. Acad. Sci. USA. 92:1013-1017.

20. Fushimi, K., et al. 1994. Functional characterization and cell immunolocalization of AQP-CD water channel in kidney collecting duct. Am. J. Physiol. 267:F573-F582.

21. Yamamoto, T., et al. 1995. Localization and expression of a collecting duct water channel, aquaporin, in hydrated and dehydrated rats. Exp. Nephrol. 3:193-201.

22. Kuwahara, M., et al. 1995. cAMP-dependent phosphorylation stimulates water permeability of aquaporin-collecting duct water channel protein expressed in Xenopus oocytes. J. Biol. Chem. 270:10384-10387.

23. Nishimoto, G., et al. 1999. Arginine vasopressin stimulates phosphorylation of aquaporin-2 in rat renal tissue. Am. J. Physiol. 276:F254-F259.

24. Katsura, T., Gustafson, C.E., Ausiello, D.A., and Brown, D. 1997. Protein kinase A phosphorylation is involved in regulated exocytosis of aquaporin-2 in transfected LLC-PK1 cells. Am. J. Physiol. 272:F817-F822.

25. Fushimi, K., Sasaki, S., and Marumo, F. 1997. Phosphorylation of serine 256 is required for cAMP-dependent regulatory exocytosis of the aquaporin-2 water channel. J. Biol. Chem. 272:14800-14804.

26. Knepper, M.A., Lankford, S.P., and Terada, Y. 1991. Renal tubular actions of ANF. Can. J. Physiol. Pharmacol. 69:1537-1545.

27. Breton, S., and Brown, D. 1998. Cold-induced microtubule disruption and relocalization of membrane proteins in kidney epithelial cells. J. Am. Soc. Nephrol. 9:155-166.

28. Gustafson, C.E., et al. 1998. Vasopressin regulated trafficking of a green fluorescent protein-aquaporin 2 chimera in LLC-PK1 cells. Histochem. Cell Biol. 110:377-386.

29. Katsura, T., et al. 1995. Constitutive and regulated membrane expression of aquaporin 1 and aquaporin 2 water channels in stably transfected LLC-PK1 epithelial cells. Proc. Natl. Acad. Sci. USA. 92:7212-7216.

30. Evan, G.I., Lewis, G.K., Ramsay, G., and Bishop, J.M. 1985. Isolation of monoclonal antibodies specific for human c-myc proto-oncogene product. Mol. Cell. Biol. 5:3610-3616.

31. Kase, H., et al. 1987. K-252 compounds, novel and potent inhibitors of protein kinase $\mathrm{C}$ and cyclic nucleotide-dependent protein kinases. Biochem. Biophys. Res. Commun. 142:436-440.

32. Katsura, T., Ausiello, D.A., and Brown, D. 1996. Direct demonstration of aquaporin-2 water channel recycling in stably transfected LLC-PK1 epithelial cells. Am. J. Pbysiol. 270:F548-F553.

33. Gustafson, C.E., et al. 2000. Recycling of AQP2 occurs through a temperature- and bafilomycin-sensitive trans-Golgi-associated compartment in LLC-PK1 cells. Am. J. Physiol. Renal Physiol. 278:F317-F326.

34. Nakamura, T., Alberola, A.M., and Granger, J.P. 1993. Role of renal inter- 
stitial pressure as a mediator of sodium retention during systemic blockade of nitric oxide. Hypertension. 21:956-960.

35. Wang, X., et al. 1998. Neuronal nitric oxide synthase is expressed in principal cell of collecting duct. Am. J. Physiol. 275:F395-F399.

36. Mundel, P., Gambaryan, S., Bachmann, S., Koesling, D., and Kriz, W. 1995. Immunolocalization of soluble guanylyl cyclase subunits in rat kidney. Histochem. Cell Biol. 103:75-79.

37. Dousa, T.P. 1999. Cyclic-3',5'-nucleotide phosphodiesterase isozymes in cell biology and pathophysiology of the kidney. Kidney Int. 55:29-62.

38. Kotera, J., Fujishige, K., and Omori, K. 2000. Immunohistochemical localization of cGMP-binding cGMP-specific phosphodiesterase (PDE5) in rat tissues. J. Histochem. Cytochem. 48:685-693.

39. Valentin, J.P., et al. 1996. Phosphodiesterase inhibitors correct resistance to natriuretic peptides in rats with Heymann Nephritis. J. Am. Soc. Nephrol. 7:582-593.

40. Sands, J.M., Nonoguchi, H., and Knepper, M.A. 1988. Hormone effects on $\mathrm{NaCl}$ permeability of rat inner medullary collecting duct. Am. J. Physiol. 255:F421-F428.

41. Nonoguchi, H., Sands, J.M., and Knepper, M.A. 1989. ANF inhibits NaCl and fluid absorption in cortical collecting duct of rat kidney. Am. J. Physiol. 256:F179-F186.

42. Schlatter, E., et al. 1996. cGMP-activating peptides do not regulate electrogenic electrolyte transport in principal cells of rat CCD. Am. J. Physiol. 271:F1158-F1165.

43. Rouch, A.J., Chen, L., Troutman, S.L., and Schafer, J.A. 1991. Na+ transport in isolated rat CCD: effects of bradykinin, ANP, clonidine, and hydrochlorothiazide. Am. J. Physiol. 260:F86-F95.

44. Hirsch, J.R., et al. 1997. Effects of sodium nitroprusside in the rat cortical collecting duct are independent of the NO pathway. Kidney Int. 51:473-476.

45. Lu, M., and Wang, W.H. 1998. Reaction of nitric oxide with superoxide inhibits basolateral $\mathrm{K}+$ channels in the rat CCD. Am. J. Physiol.
275:C309-C316

46. Nonoguchi, H., Knepper, M.A., and Manganiello, V.C. 1987. Effects of atrial natriuretic factor on cyclic guanosine monophosphate and cyclic adenosine monophosphate accumulation in microdissected nephron segments from rats. J. Clin. Invest. 79:500-507.

47. Marples, D., et al. 1998. Dynein and dynactin colocalize with AQP2 water channels in intracellular vesicles from kidney collecting duct. Am. J. Physiol. 274:F384-F394.

48. Berl, T., and Robertson, G.L. 2000. Pathophysiology of water metabolism. In The kidney. B.M. Brenner, editor. W.B. Saunders Co. Philadelphia, Pennsylvania, USA. 866-924.

49. Hochberg, Z., Even, L., and Danon, A. 1998. Amelioration of polyuria in nephrogenic diabetes insipidus due to aquaporin-2 deficiency. Clin. Endocrinol. (Oxf.) 49:39-44.

50. Chou, C.L., DiGiovanni, S.R., Luther, A., Lolait, S.J., and Knepper, M.A. 1995. Oxytocin as an antidiuretic hormone. II. Role of V2 vasopressin receptor. Am. J. Physiol. 269:F78-F85.

51. Lencer, W.I., Brown, D., Ausiello, D.A., and Verkman, A.S. 1990. Endocytosis of water channels in rat kidney: cell specificity and correlation with in vivo antidiuresis. Am. J. Physiol. 259:C920-C932.

52. Edwards, B.R., Gellai, M., and Valtin, H. 1980. Concentration of urine in the absence of ADH with minimal or no decrease in GFR. Am. J. Physiol. 239:F84-F91.

53. Piccinni, Z., and Parisi, M. 1973. Effect of guanosine $3^{\prime}: 5^{\prime}$-monophosphate on the hydroosmotic activity of adenosine $3^{\prime}: 5^{\prime}$-monophosphate in toad urinary bladder. Biochim. Biophys. Acta. 311:625-629.

54. Bourgoignie, J., Guggenheim, S., Kipnis, D., and Klahr, S. 1969. Cyclic guanosine monophosphate: effects on short-circuit current and water permeability. Science. 165:1362-1363.

55. Bentley, P.J. 1970. Urinary bladder of leopard frogs (Rana pipiens): effects of vasotocin, cyclic AMP, cyclic GMP and aldosterone. Endocrinology. 87:1367-1368 